\title{
Spiral Plaque on the Left Ankle
}

\author{
Sherman Chu, DO; Luisa F. Christensen, MD; Kord Honda, MD; Kevin D. Cooper, MD
}

\section{Eligible for 1 MOC SA Credit From the ABD}

This Photo Challenge in our print edition is eligible for 1 self-assessment credit for Maintenance of Certification from the American Board of Dermatology (ABD). After completing this activity, diplomates can visit the ABD website (http://www.abderm.org) to self-report the credits under the activity title "Cutis Photo Challenge." You may report the credit after each activity is completed or after accumulating multiple credits.

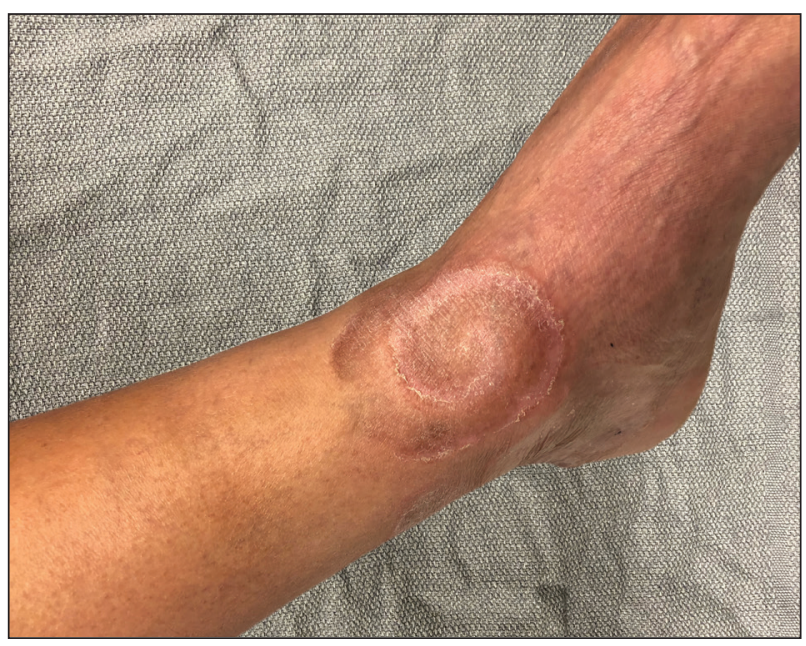
mycosis fungoides with several hyperpigmented patches at sites of prior involvement on the trunk and upper and lower extremities. No cervical, axillary, or inguinal lymphadenopathy was noted. A 4-mm punch biopsy was performed and sent for histopathologic examination.

\section{WHAT'S YOUR DIAGNOSIS?}
a. erythema annulare centrifugum
b. erythema gyratum repens
c. granuloma annulare
d. recurrent cutaneous T-cell lymphoma
e. tinea pedis

A 60-year-old man presented with a whorl-like plaque on the left ankle that he had noticed while undergoing treatment with narrowband UB every other week and nitrogen mustard gel daily for stage IB cutaneous T-cell lymphoma, or changes in diet. His medical history included multiple sclerosis, vascular disease, and stroke. Physical examination revealed an $8 \times 6-\mathrm{cm}$, welldemarcated, slightly scaly, erythematous plaque with a spiral appearance and peripheral hyperpigmentation involving the left ankle. The remainder$$
\text { e. tinea pedis }
$$

PLEASE TURN TO PAGE 149 FOR THE DIAGNOSIS

From the Department of Dermatology, University Hospitals Cleveland Medical Center, Ohio. Drs. Chu and Cooper also are from the Department of Dermatology, Case Western Reserve University, Cleveland. Dr. Chu also is from University Hospitals Regional Hospitals, Richmond Heights, Ohio. The authors report no conflict of interest.

Correspondence: Sherman Chu, DO, Department of Dermatology, Case Western Reserve University, 2109 Adelbert Rd, Cleveland, OH (shermac619@gmail.com).

doi:10.12788/cutis.0336 


\section{THE DIAGNOSIS:}

\section{Recurrent Cutaneous T-Cell Lymphoma}

T he skin biopsy revealed alternating orthokeratosis and parakeratosis with mild to moderate spongiosis and intraepidermal vesiculation as well as individual and nested atypical mononuclear cells with moderately enlarged hyperchromatic nuclei in the epidermis. There was a superficial interstitial lymphocytic infiltrate with occasional enlarged cells (Figure, A and B), and atypical cells in the epidermis and dermis stained with antibodies against CD3 and CD4 (Figure, $\mathrm{C}$ and D) but not against CD20 or CD8. These histopathologic findings were consistent with cutaneous T-cell lymphoma (CTCL), mycosis fungoides (MF) type. Additional application of bexarotene gel on days the patient received narrowband UVB was recommended with noted improvement of the skin.

Cutaneous T-cell lymphomas are a heterogenous group of diseases with monoclonal proliferation of $\mathrm{T}$ lymphocytes that largely are confined to the skin at the time of diagnosis. ${ }^{1}$ The incidence of CTCL rose steadily for more than 25 years, with an annual age-adjusted incidence of 6.4 to 9.6 cases per million individuals in the United States from 1973 to 2002. ${ }^{2}$ Mycosis fungoides is the most common classification of CTCL. It usually is characterized by patches or plaques of scaly erythema or poikiloderma; however, it also can present with annular, arcuate, concentrative, annular and linear morphologies. Mycosis fungoides tumor cells typically express a mature memory $\mathrm{T}$ helper cell phenotype of $\mathrm{CD}^{+}, \mathrm{CD}^{+}$, and CD8-, but there are different variants that have been discovered. ${ }^{3}$ Mycosis fungoides distributed in a spiral pattern is a distinctly unusual manifestation. Mechanisms of such dynamic morphologies are unknown but may represent an interplay between malignant cell proliferation and lost immune responses in temporospatial relationships.

The presence of keratotic gyrate lesions on acral surfaces should raise the possibility of pagetoid reticulosis. However, our patient had a history of MF involving areas of the body beyond the extremities, making this diagnosis less likely. Pagetoid reticulosis is categorized as an MF variant under the current World Health OrganizationEuropean Organization for Research and Treatment of
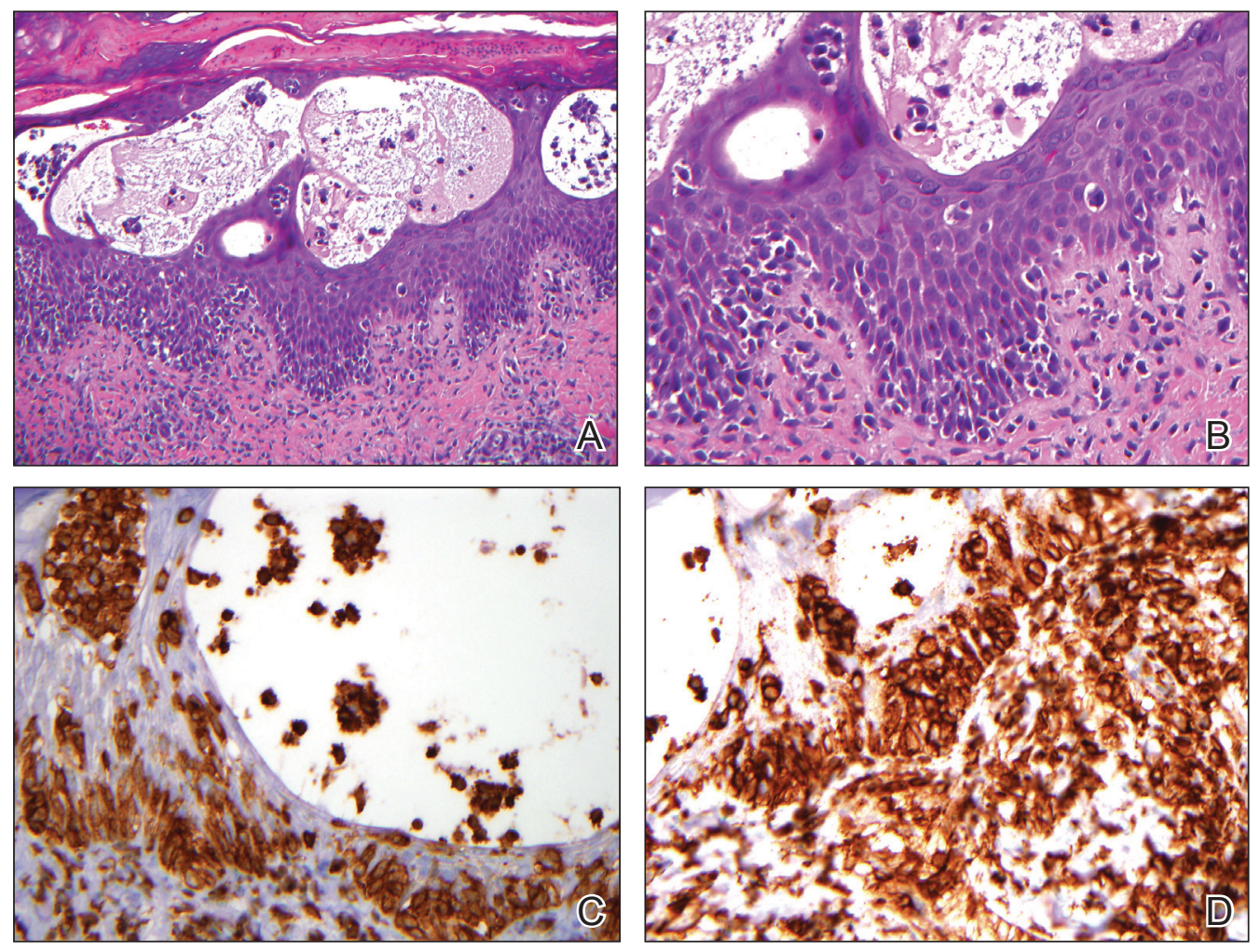

Histopathology of cutaneous T-cell lymphoma. A, Mild to moderate spongiosis and intraepidermal vesiculation with individual and nested atypical mononuclear cells (H\&E, original magnification $\times 20)$. B, Moderately enlarged hyperchromatic nuclei in the epidermis and superficial interstitial lymphocytic infiltrate with occasional enlarged cells (H\&E, original magnification $\times 40$ ). C and D, Immunostaining showed $\mathrm{CD}^{+}$and $\mathrm{CD} 4^{+}$atypical cells in the epidermis and dermis, respectively (original magnifications $\times 40$ ). 
Cancer classification for cutaneous lymphomas. ${ }^{4}$ Pagetoid reticulosis clinically presents as a solitary psoriasiform or hyperkeratotic patch or plaque that affects the distal extremities. Variable immunophenotypes have been shown in pagetoid reticulosis, such as $\mathrm{CD} 4^{-} / \mathrm{CD} 8^{+}$and $\mathrm{CD}^{-/} / \mathrm{CD}^{-}$, while classic MF typically shows $\mathrm{CD}^{+} / \mathrm{CD}^{-}$, as in our case. ${ }^{5}$

Tinea pedis is a superficial fungal infection usually caused by anthropophilic dermatophytes, with Trichophyton rubrum being the most common organism. Four common clinical presentations of tinea pedis have been identified: interdigital, moccasin, vesicular, and acute ulcerative. Clinical presentation ranges from macerations, ulcerations, and erosions in the toe web spaces to dry hyperkeratotic scaling and fissures on the plantar foot. ${ }^{6}$ Tinea pedis primarily affects the plantar and interdigital spaces, sparing the dorsal foot and ankle. Treatment is recommended to alleviate symptoms and limit the spread of infection; topical antifungals for 4 weeks is the treatment of choice. However, recurrence is common, and maintenance therapy often is indicated. Oral antifungals or a combination of both topical and oral medications may be needed in certain cases. ${ }^{7}$

Erythema annulare centrifugum (EAC) is a rare dermatologic disease described as erythematous or urticarial papules that can enlarge centrifugally to form annular lesions that clear centrally. Thought to be a hypersensitivity reaction to an underlying condition, EAC has been associated with fungal infections, various cutaneous diseases, and even internal malignancies. Clinically, EAC can be divided into 2 forms: deep and superficial. Deep gyrate erythema is characterized by a firm indurated border with rare scaling and pruritus that histologically shows perivascular lymphocytic infiltration in the upper and deep dermis. Superficial gyrate erythema has minimally elevated lesions with an indistinct border and trailing scales and pruritus; histopathologic findings present a dense, perivascular, lymphocytic infiltration restricted to the upper dermis. ${ }^{8}$ Therapy for EAC is directed at relieving symptoms and treating the underlying condition if there is one associated.

Granuloma annulare (GA) is a common skin disorder classically characterized by ringed erythematous plaques, though many variants have been identified. Localized GA is the most common variant and presents with pink-red, nonscaly, annular patches or plaques, typically affecting the hands and feet. Generalized GA is characterized as diffuse annular patches or plaques classically affecting the trunk and extremities. Histology is notable for mucin with a palisading or interstitial pattern of granulomatous inflammation, which was not evident in our patient. ${ }^{9}$ Topical or intralesional corticosteroids are the first-line treatment of localized GA; however, localized GA generally is self-limited, and treatment often is not necessary. Treatment with cryosurgery, laser therapy, and topical dapsone and tacrolimus also has been described, but evidence of the efficacy of these agents is limited. For generalized
GA, phototherapy currently is the most reliable therapy. Systemic therapies include antimalarials, fumaric acid esters, biologics, antimicrobials, and isotretinoin. ${ }^{10}$

Erythema gyratum repens (EGR) is a rare dermatologic disease described as erythematous concentric bands arranged in parallel rings that can be annular, figurate, or gyrate, with a fine scale trailing the leading edge. Histopathologic features of EGR are nonspecific but are characterized by a perivascular, superficial, mononuclear dermatitis. Diagnosis is based on its characteristic clinical presentation. Although EGR commonly is associated with internal malignancies such as bronchial carcinoma, it also may be associated with benign conditions. ${ }^{11}$ Improvement often is seen with successful therapy of the underlying associated malignancy. ${ }^{12}$

Treatment of MF is based on tumor-node-metastasisblood classification, prognostic factors, and clinical stage at the time of diagnosis. Early-stage MF (IA-IIA) commonly is treated with skin-directed therapies such as topical corticosteroids, topical mechlorethamine, topical retinoids, UV phototherapy, and localized radiotherapy. In late stages (IIB-IV), systemic therapy is indicated and includes systemic retinoids, interferon alfa, chemotherapy, monoclonal antibodies, and psoralen plus UVA. ${ }^{13}$ In many cases, patients may require combination therapy to achieve remission or better control of their condition, as in our patient.

\section{REFERENCES}

1. Pulitzer M. Cutaneous T-cell lymphoma. Clin Lab Med. 2017;37:527-546.

2. Jawed SI, Myskowski PL, Horwitz S, et al. Primary cutaneous T-cell lymphoma (mycosis fungoides and Sézary syndrome): part I. diagnosis: clinical and histopathologic features and new molecular and biologic markers. J Am Acad Dermatol. 2014;70:205.e1-16; quiz 221-222.

3. Virmani P, Myskowski PL, Pulitzer M. Unusual variants of mycosis fungoides. Diagn Histopathol (Oxf). 2016;22:142-151.

4. Willemze R, Cerroni L, Kempf W, et al. The 2018 update of the WHO-EORTC classification for primary cutaneous lymphomas. Blood. 2019;133:1703-1714.

5. Haghighi B, Smoller BR, LeBoit PE, et al. Pagetoid reticulosis (Woringer-Kolopp disease): an immunophenotypic, molecular, and clinicopathologic study. Mod Pathol. 2000;13:502-510.

6. Canavan TN, Elewski BE. Identifying signs of tinea pedis: a key to understanding clinical variables. J Drugs Dermatol. 2015;14 (10 suppl):S42-S47.

7. Ilkit M, Durdu M. Tinea pedis: the etiology and global epidemiology of a common fungal infection. Crit Rev Microbiol. 2015;41:374-388.

8. Weyers W, Diaz-Cascajo C, Weyers I. Erythema annulare centrifugum: results of a clinicopathologic study of 73 patients. Am J Dermatopathol. 2003;25:451-462.

9. Piette EW, Rosenbach M. Granuloma annulare: clinical and histologic variants, epidemiology, and genetics. J Am Acad Dermatol. 2016;75:457-465

10. Wang J, Khachemoune A. Granuloma annulare: a focused review of therapeutic options. Am J Clin Dermatol. 2018;19:333-344.

11. Rongioletti F, Fausti V, Parodi A. Erythema gyratum repens is not an obligate paraneoplastic disease: a systematic review of the literature and personal experience. J Eur Acad Dermatol Venereol. 2014;28:112-115.

12. Boyd AS, Neldner KH, Menter A. Erythema gyratum repens: a paraneoplastic eruption. J Am Acad Dermatol. 1992;26(5, pt 1):757-762.

13. Trautinger F, Eder J, Assaf C, et al. European Organisation for Research and Treatment of Cancer consensus recommendations for the treatment of mycosis fungoides/Sézary syndrome-update 2017. Eur J Cancer. 2017;77:57-74. 